\title{
Spectral mapping inclusions for the Phillips functional calculus in Banach spaces and algebras
}

\author{
by
}

\author{
Eva Fašangová (Praha) and Pedro J. Miana (Zaragoza)
}

Abstract. We investigate the weak spectral mapping property (WSMP)

$$
\overline{\widehat{\mu}(\sigma(A))}=\sigma(\widehat{\mu}(A)),
$$

where $A$ is the generator of a $\mathcal{C}_{0}$-semigroup in a Banach space $X, \mu$ is a measure, and $\widehat{\mu}(A)$ is defined by the Phillips functional calculus. We consider the special case when $X$ is a Banach algebra and the operators $e^{A t}, t \geq 0$, are multipliers.

1. Introduction. Let $X$ be a Banach space. We denote by $\mathcal{B}(X)$ the space of bounded linear operators on $X$ and by $\left(e^{A t}\right)_{t \geq 0} \subset \mathcal{B}(X)$ a $\mathcal{C}_{0^{-}}$ semigroup with infinitesimal generator $A$. Suppose that the growth of the semigroup can be controlled as $\left\|e^{A t}\right\| \leq c w(t)$, where $w:[0, \infty) \rightarrow[0, \infty)$ is measurable, $w(t+s) \leq w(t) w(s), w(0)=1, c>0$ is a constant. Define $\varrho_{w}:=\lim _{t \rightarrow \infty} w(t)^{1 / t}\left(=\inf _{t>0} w(t)^{1 / t}\right)$. Let $M\left(\mathbb{R}_{+}, w\right)$ be the space of all complex Borel measures $\mu$ on $\mathbb{R}_{+}$such that

$$
\int_{0}^{\infty} w(t) d|\mu|(t)<\infty .
$$

We will denote by $\widehat{\mu}(\lambda):=\int_{0}^{\infty} e^{\lambda t} d \mu(t)$ the Laplace transform of $\mu$. The Phillips functional calculus can be expressed as a continuous algebra homomorphism:

$$
\begin{gathered}
\mu \in\left(M\left(\mathbb{R}_{+}, w\right), *\right) \mapsto \widehat{\mu}(A) \in(\mathcal{B}(X), \circ), \\
\widehat{\mu}(A) x:=\int_{0}^{\infty} e^{A t} x d \mu(t), \quad x \in X .
\end{gathered}
$$

We have the following basic examples: if $\mu:=\delta_{t_{0}}$ for $t_{0} \geq 0$, then $\widehat{\mu}(A)$ $=e^{A t_{0}}, \widehat{\mu}(\lambda)=e^{\lambda t_{0}}$; if $\mu(t):=e^{-\lambda_{0} t} d t$ for $\operatorname{Re} \lambda_{0}>\ln \varrho_{w}$, then $\widehat{\mu}(A)=$

2000 Mathematics Subject Classification: 47A60, 47A10, 46H30.

This work was partially supported by grants GA ČR 201/01/D094, MSM 113200007 of the Czech Ministry of Education, the Spanish DGES BFM2001-1793 of the MCYTDGI and FEDER and Programa Europa, CAI, 2002. 
$\left(\lambda_{0}-A\right)^{-1}, \widehat{\mu}(\lambda)=\left(\lambda_{0}-\lambda\right)^{-1}$. From the latter we obtain $\sigma(A) \subset\{\operatorname{Re} \lambda \leq$ $\left.\ln \varrho_{w}\right\}$. Note that $\widehat{\mu}$ is a bounded function on the halfspace $\left\{\operatorname{Re} \lambda \leq \ln \varrho_{w}\right\}$, holomorphic in the interior, so it is defined on $\sigma(A)$. In particular, if $A$ is a generator of a $\mathcal{C}_{0}$-group, we can even take measures on $\mathbb{R}$ and the integral in (1) from $-\infty$ to $\infty$. Details may be found in [4, Chapter XV].

It is well known that the weak spectral mapping property (WSMP),

$$
\overline{\widehat{\mu}(\sigma(A))}=\sigma(\widehat{\mu}(A)),
$$

holds only in some special cases (for resolvents: $\widehat{\mu}(\cdot)=1 /\left(\lambda_{0}-\cdot\right)$ ) for analytic semigroups for $\widehat{\mu}(\cdot)=e^{t_{0}} \cdot([3, \mathrm{p} .281])$; if $A$ is a generator of a $\mathcal{C}_{0}$-group with non-quasianalytic growth and the singular part of the measure $\mu$ is zero $([1],[6]))$. On the other hand, there are examples of $\mathcal{C}_{0}$-semigroups where the right to left inclusion is not true ([3, p. 271]).

In this paper we investigate the two inclusions separately. The left to right inclusion is well known (see [4, Theorem 16.3.5]):

$$
\overline{\widehat{\mu}(\sigma(A))} \subset \sigma(\widehat{\mu}(A)) \text {. }
$$

Nevertheless, we present a straightforward proof.

The opposite inclusion is investigated in the context of Banach algebras. Under the condition that $e^{A t}, t \geq 0$, are multipliers on the Banach algebra $X$, we prove the inclusion

$$
\widehat{\mu}(A)(\Delta X) \subset \widehat{\mu}(\sigma(A))
$$

(note that by definition $\widehat{\mu}(A)(\Delta X) \subset \sigma(\widehat{\mu}(A)))$. The proof is based on the fact that WSMP holds for the resolvents and uses the Gelfand theory.

Finally, we present some examples where WSMP can be obtained from the above two inclusions: if $\widehat{\mu}(A)$ has natural spectrum, then WSMP is true. In particular, this is the case for semigroups in the Banach algebra $X$ itself, like the Gaussian, Poisson or fractional integral semigroups. We also present the basic example of the shift semigroup on $L^{1}\left(\mathbb{R}_{+}\right)$and the shift group on $L^{1}(\mathbb{R})$, showing that in general we cannot expect equality. In particular, WSMP does not hold for $\mu$ whenever the Wiener-Pitt phenomenon holds for $\mu$.

\section{Preliminaries}

2.1. The first inclusion. In this subsection $X$ is a Banach space, $\left(e^{A t}\right)_{t \geq 0}$ $\subset \mathcal{B}(X)$ is a $\mathcal{C}_{0}$-semigroup with growth function $w$ and generator $A$. We present a shorter proof of the (well known) spectral inclusion theorem (see e.g. [4]). The elementary facts used in the proof may be found in [3, Lemma II.1.3, Section IV.1].

Proposition 2.1. If $\mu \in M\left(\mathbb{R}_{+}, w\right)$ then

$$
\widehat{\mu}(\sigma(A)) \subset \sigma(\widehat{\mu}(A)) .
$$


Proof. Let $\lambda \in \sigma(A)$. Then $\operatorname{Re} \lambda \leq \ln \varrho_{w}$, as noticed before. We will use the following formula (for $\lambda \in \mathbb{C}, t \geq 0$ ):

$$
e^{\lambda t} x-e^{A t} x= \begin{cases}(\lambda-A) \int_{0}^{t} e^{\lambda(t-s)} e^{A s} x d s, & x \in X, \\ \int_{0}^{t} e^{\lambda(t-s)} e^{A s}(\lambda-A) x d s, & x \in D(A) .\end{cases}
$$

We have (the integral converges provided $\operatorname{Re} \lambda \leq \ln \varrho_{w}$ )

$$
\widehat{\mu}(\lambda) x-\widehat{\mu}(A) x=\int_{0}^{\infty}\left[e^{\lambda t} x-e^{A t} x\right] d \mu(t), \quad x \in X .
$$

This shows that if $\lambda \in \sigma_{\text {ap }}(A)$ then $\widehat{\mu}(\lambda) \in \sigma_{\text {ap }}(\widehat{\mu}(A))$ : in fact, let $\lambda \in \sigma_{\text {ap }}(A)$ with approximate eigenvector $\left(x_{n}\right),\left\|x_{n}\right\|=1$; then for a fixed $t$ we have $e^{\lambda t} x_{n}-e^{A t} x_{n} \rightarrow 0$ and using the Lebesgue dominated convergence theorem we find that $\widehat{\mu}(\lambda) x_{n}-\widehat{\mu}(A) x_{n} \rightarrow 0$, i.e. $\widehat{\mu}(\lambda) \in \sigma_{\text {ap }}(\widehat{\mu}(A))$.

On the other hand, if $\lambda \in \sigma(A) \backslash \sigma_{\text {ap }}(A)$, i.e. the range $\operatorname{rg}(\lambda-A)$ is not dense, then there exists an $x^{*} \in X^{*}$ such that $\left\|x^{*}\right\|=1$ and $x^{*}(\operatorname{rg}(\lambda-A))=0$. Inserting the first expression in (2) into (3) and applying $x^{*}$ we get

$$
x^{*}(\widehat{\mu}(\lambda) x-\widehat{\mu}(A) x)=\int_{0}^{\infty} x^{*}\left((\lambda-A) \int_{0}^{t} e^{\lambda(t-s)} e^{A s} x d s\right) d \mu(t)=0 .
$$

This shows that $\operatorname{rg}(\widehat{\mu}(\lambda)-\widehat{\mu}(A))$ is not dense, i.e. $\widehat{\mu}(\lambda) \in \sigma(\widehat{\mu}(A))$.

2.2. Notions in Banach algebras. We collect the basic definitions needed to formulate the result of Section 3. For more details see e.g. [5, Sections 4.1, 4.6].

Let $X$ be a commutative Banach algebra. We denote by $\Delta X:=\{\varphi \in$ $X^{\prime} ; 0 \neq \varphi$ is multiplicative $\}$ the Gelfand space of $X$ equipped with the $w^{*}$ topology, by $\sigma_{X}(a)$ the spectrum of $a \in X$, by $\varrho(a):=\mathbb{C} \backslash \sigma_{X}(a)$ the resolvent set and by $\widehat{a}(\varphi):=\varphi(a)$ the Gelfand transform of $a$. The multiplier algebra of $X$ is $M(X):=\{L \in \mathcal{B}(X) ; a L b=(L a) b, a, b \in X\}$ with composition product. For $a \in X$ set $L_{a} b:=a b$; then $L_{a} \in M(X)$. We say that $X$ is without order if $a \mapsto L_{a}$ is injective. An algebra $A$ is called semisimple if $a \mapsto \widehat{a}$ is injective.

We have the following properties:

- $\sigma_{X}(a)=\overline{\widehat{a}(\Delta X)}$;

- if $X$ is without order then $M(X)$ is a closed, full, commutative subalgebra of $\mathcal{B}(X)$, and $\Delta X$ is canonically embedded into $\Delta M(X)$ : $\varphi \in \Delta X \mapsto \varphi_{1}, \varphi_{1}(L):=\varphi(L a) / \varphi(a)$, where $a$ is such that $\varphi(a) \neq 0$;

- if $A$ is semisimple then $A$ is without order. 
Therefore, when $X$ is without order, for any $L \in M(X)$ we have

$$
\widehat{L}(\Delta X) \subset \widehat{L}(\Delta M(X)), \quad \overline{\widehat{L}(\Delta M(X))}=\sigma_{M(X)}(L)=\sigma_{\mathcal{B}(X)}(L) .
$$

We say that $L \in M(X)$ has natural spectrum if

$$
\overline{\widehat{L}(\Delta X)}=\sigma_{\mathcal{B}(X)}(L) .
$$

3. Second inclusion. The setting of this section is the following: $X$ is a commutative Banach algebra without order, $\left(e^{A t}\right)_{t \geq 0}$ is a $\mathcal{C}_{0}$-semigroup in $\mathcal{B}(X)$, with infinitesimal generator $A$, such that $e^{A t} \in M(X)$ for any $t \geq 0$, and $w$ is a corresponding function controlling the growth.

Lemma 3.1. If $\lambda \in \varrho(A)$ then $(\lambda-A)^{-1} \in M(X)$. If $\mu \in M\left(\mathbb{R}_{+}, w\right)$ then $\widehat{\mu}(A) \in M(X)$.

Proof. If $x \in X$ and $y \in D(A)$ then $x y \in D(A)$ and $A(x y)=x(A y)$, as can be easily checked from the definition of $A$. Set for shorthand $B:=$ $(\lambda-A)^{-1} \in \mathcal{B}(X)$. To see that $B \in M(X)$, let $y_{1}:=B\left(x_{1} x_{2}\right), y_{2}:=B x_{2}$; then

$$
x_{1} x_{2}=x_{1}(\lambda-A) y_{2}=\lambda x_{1} y_{2}-x_{1} A y_{2}=(\lambda-A)\left(x_{1} y_{2}\right),
$$

hence $B\left(x_{1} x_{2}\right)=x_{1} y_{2}$. Since $e^{A t} \in M(X), t \geq 0$, we infer trivially from the definition that $\widehat{\mu}(A) \in M(X)$.

Theorem 3.2. Let $X, e^{A t}, w$ be as above. Then for any $\mu \in M\left(\mathbb{R}_{+}, w\right)$ we have

$$
\widehat{\mu}(A)(\Delta X) \subset \widehat{\mu}(\sigma(A)) .
$$

Proof. Let $\varphi \in \Delta X$. Take $x \in X$ such that $\varphi(x) \neq 0$. Let $\varphi_{1} \in \Delta M(X)$ be the unique element associated to $\varphi$ by the canonical embedding.

Since $t \in[0, \infty) \mapsto e^{A t} x \in X$ is continuous, the map $t \mapsto \varphi\left(e^{A t} x\right)=$ $\varphi_{1}\left(e^{A t}\right) \varphi(x)$ is continuous, and hence $t \mapsto \varphi_{1}\left(e^{A t}\right)$ is continuous as well. From the functional equation $\varphi_{1}\left(e^{A(t+s)}\right)=\varphi_{1}\left(e^{A t} e^{A s}\right)=\varphi_{1}\left(e^{A t}\right) \varphi_{1}\left(e^{A s}\right)$, $\varphi_{1}\left(e^{A 0}\right)=\varphi_{1}(I)=1$ we get the existence of some $z \in \mathbb{C}$ such that $\varphi_{1}\left(e^{A t}\right)=$ $e^{z t}$ for all $t \geq 0$.

Fix a $\lambda$ such that $\operatorname{Re} \lambda>\ln \varrho_{w}$. Then we may apply $\varphi$ to the equality $(\lambda-A)^{-1} x=\int_{0}^{\infty} e^{A t} x e^{-\lambda t} d t$ to get

$$
\begin{aligned}
\varphi_{1}\left((\lambda-A)^{-1}\right) \varphi(x) & =\varphi\left((\lambda-A)^{-1} x\right)=\int_{0}^{\infty} \varphi\left(e^{A t} x\right) e^{-\lambda t} d t \\
& =\int_{0}^{\infty} e^{z t} e^{-\lambda t} d t \varphi(x)=\frac{1}{\lambda-z} \varphi(x) \neq 0 .
\end{aligned}
$$

After dividing by $\varphi(x)$ and using the spectral mapping theorem for the resolvent (see [3, Theorem IV.1.13]) we get 


$$
\begin{array}{r}
\frac{1}{\lambda-z}=\varphi_{1}\left((\lambda-A)^{-1}\right) \in \widehat{(\lambda-A)^{-1}}(\Delta M(X)) \backslash\{0\}=\sigma\left((\lambda-A)^{-1}\right) \backslash\{0\} \\
=\left\{\frac{1}{\lambda-\nu} ; \nu \in \sigma(A)\right\}=: \frac{1}{\lambda-\sigma(A)},
\end{array}
$$

hence $z \in \sigma(A)$.

With the same procedure, applying $\varphi_{1}$ to both sides of (1) we get

$$
\begin{aligned}
\varphi_{1}(\widehat{\mu}(A)) \varphi(x) & =\varphi(\widehat{\mu}(A) x)=\int_{0}^{\infty} \varphi\left(e^{A t} x\right) d \mu(t) \\
& =\int_{0}^{\infty} e^{z t} d \mu(t) \varphi(x)=\widehat{\mu}(z) \varphi(x),
\end{aligned}
$$

and hence

$$
\widehat{\mu}(A)(\varphi)=\varphi_{1}(\widehat{\mu}(A))=\widehat{\mu}(z) \in \widehat{\mu}(\sigma(A)) .
$$

REMARK. The idea of the above proof is inspired by [4, Sections 16.3, 16.4] (see also [2, Theorems 2.16, 2.19]). There, another subalgebra of $\mathcal{B}(X)$ is considered (the commutative algebra generated by the resolvents $\left.(\lambda-A)^{-1}\right)$ and an element of its Gelfand space is applied to functional equations (semigroup law, resolvent equation, integral representation of $\widehat{\mu}(A)$ ).

4. Applications. By now we have proved that in the context of a semigroup of multipliers on a Banach algebra we have

$$
\widehat{\mu}(A)(\Delta X) \subset \widehat{\mu}(\sigma(A)) \subset \sigma(\widehat{\mu}(A))=\widehat{\mu}(A)(\Delta M(X)) ;
$$

recall that $\Delta X \subset \Delta M(X)$. From the definition of natural spectrum we obtain the following result.

Corollary 4.1. Let $X, e^{A t}, w$ be as in the previous section, and $\mu \in$ $M\left(\mathbb{R}_{+}, w\right)$. If $\widehat{\mu}(A)$ has natural spectrum, then WSMP holds, i.e.

$$
\overline{\widehat{\mu}(\sigma(A))}=\overline{\widehat{\widehat{\mu}(A)}(\Delta X)}=\sigma(\widehat{\mu}(A)) .
$$

4.1. Semigroups in Banach algebras. We call a set $\left\{a^{t} ; t>0\right\} \subset X$ a semigroup in the commutative Banach algebra $X$ if $a^{t+s}=a^{t} a^{s}, t, s>0$, and $\lim _{t \rightarrow 0_{+}} a^{t} b=b$ for any $b \in X$. It is easy to check that then $\left\{L_{a^{t}} ; t>0\right\} \cup$ $\{I\} \subset \mathcal{B}(X)$ is a $\mathcal{C}_{0}$-semigroup of operators. We denote by $A$ its infinitesimal generator, i.e. $e^{A t}=L_{a^{t}}$. Let $w$ be as in the introduction.

Proposition 4.2. Let $X$ be a commutative semisimple Banach algebra without identity, and $\left\{a^{t} ; t>0\right\}$ a semigroup in $X$.

- WSMP holds for $\delta$-measures, i.e. when $\widehat{\mu}(\cdot)=e^{t}$ :

$$
\overline{e^{t \sigma(A)}}=\sigma\left(e^{A t}\right), \quad t>0 .
$$


- If $t \in(0, \infty) \mapsto a^{t} \in X$ is continuous, then WSMP holds for the Phillips calculus, i.e.

$$
\overline{\widehat{\mu}(\sigma(A))}=\sigma(\widehat{\mu}(A)), \quad \mu \in M\left(\mathbb{R}_{+}, w\right) .
$$

Proof. By [5, pp. 365, 369] the multiplier $L_{b}$ has natural spectrum for any $b \in X$, so we may apply Corollary 4.1 to $\mu=\delta_{t}$.

If in addition $t \mapsto a^{t}$ is continuous, then the integral $\int_{0}^{\infty} a^{t} d \mu(t)=: a_{\mu}$ exists in $X$, meaning that $\widehat{\mu}(A)=L_{a_{\mu}}$, which has natural spectrum.

EXAmple 1. $X:=\left(L^{1}\left(\mathbb{R}^{n}\right), *\right)$ is a commutative semisimple Banach algebra without identity. The multiplier algebra is isomorphic to $\left(M\left(\mathbb{R}^{n}\right), *\right)$. The Gaussian semigroup and the Poisson semigroup

$$
\begin{aligned}
g^{t}(x) & :=\frac{1}{(4 \pi t)^{n / 2}} e^{-|x|^{2} / 4 t}, \\
p^{t}(x) & :=\Gamma\left(\frac{n+1}{2}\right) \frac{1}{\pi^{(n+1) / 2}} \frac{t}{\left(t^{2}+|x|^{2}\right)^{(n+1) / 2}}, \quad x \in \mathbb{R}^{n}, t>0,
\end{aligned}
$$

are semigroups in $X$, they are bounded by 1 . Since the mappings $(0, \infty) \ni$ $t \mapsto g^{t}$ and $(0, \infty) \ni t \mapsto p^{t}$ are continuous in $X$, WSMP holds for their Phillips calculus.

Example 2. $X:=\left(L^{1}\left(\mathbb{R}_{+}\right), *\right)$ is a commutative semisimple Banach algebra without identity. The multiplier algebra is isomorphic to $\left(M\left(\mathbb{R}_{+}\right), *\right)$. The fractional integral semigroup

$$
I^{t}(x):=\frac{1}{\Gamma(t)} x^{t-1} e^{-x}, \quad x, t>0,
$$

is a semigroup in $X$, it is bounded by $1,(0, \infty) \ni t \mapsto I^{t}$ is continuous in $X$, hence WSMP holds for its Phillips calculus.

Since these semigroups are even holomorphic in a sector containing $(0, \infty)$, it is well known that WSMP holds for the Dirac measures $\mu=\delta_{t_{0}}$. See [3] and [7] for more details.

4.2. The shift semigroup. Let us consider the semisimple commutative Banach algebra $X:=\left(L^{1}\left(\mathbb{R}_{+}\right), *\right)$. Its Gelfand space is isomorphic to $\overline{\mathbb{C}}_{-}:=$ $\{\lambda \in \mathbb{C} ; \operatorname{Re} \lambda \leq 0\}$ (via Laplace transform), and its multiplier algebra is isomorphic to $\left(M\left(\mathbb{R}_{+}\right), *\right)$. Consider the shift semigroup with its generator:

$$
\begin{gathered}
A f=-f^{\prime}, \quad D(A)=\left\{f \in L^{1}\left(\mathbb{R}_{+}\right) ; f^{\prime} \in L^{1}\left(\mathbb{R}_{+}\right), f(0)=0\right\}, \\
\left(e^{A t} f\right)(s):= \begin{cases}f(s-t), & s \geq t, \\
0, & 0<s<t .\end{cases}
\end{gathered}
$$

One has $\left\|e^{A t}\right\|=1$, so we take $w(t)=1$ for $t \geq 0$. The Phillips calculus is

$$
(\widehat{\mu}(A) f)(s)=\int_{0}^{\infty} e^{A t} f(s) d \mu(t)=\int_{0}^{s} f(s-t) d \mu(t)=(\mu * f)(s)=\left(L_{\mu} f\right)(s) .
$$


One can directly compute:

so

$$
\sigma(A)=\overline{\mathbb{C}}_{-}, \quad \sigma\left(e^{A t}\right)=\{\lambda \in \mathbb{C} ;|\lambda| \leq 1\},
$$

$$
\begin{aligned}
& \overline{e^{t \sigma(A)}}=e^{t \sigma(A)} \cup\{0\}=\sigma\left(e^{A t}\right), \\
& \sigma(\widehat{\mu}(A))=\sigma\left(L_{\mu}\right), \\
& \widehat{\mu}(\sigma(A))=\widehat{\mu}\left(\overline{\mathbb{C}}_{-}\right)=\widehat{\mu}(\Delta X) .
\end{aligned}
$$

This shows that for $\mu \in M\left(\mathbb{R}_{+}\right)$, WSMP holds if and only if $\mu$ has natural spectrum. So whenever for a measure $\mu$ the Wiener-Pitt phenomenon holds (meaning that $\mu$ is not invertible in $M\left(\mathbb{R}_{+}\right)$despite inf $\left|\widehat{\mu}\left(\overline{\mathbb{C}}_{-}\right)\right|>0$ ), WSMP does not hold for this $\mu$. The existence of such measures is shown in [8].

4.3. The shift group. Let $X:=\left(L^{1}(\mathbb{R}), *\right)$. Then the Gelfand space is isomorphic to $i \mathbb{R}$ and the multiplier algebra is isomorphic to $(M(\mathbb{R}), *)$. Consider the shift group with its generator:

$$
\begin{gathered}
A f=-f^{\prime}, \quad D(A)=\left\{f \in L^{1}(\mathbb{R}) ; f^{\prime} \in L^{1}(\mathbb{R})\right\}, \\
\left(e^{A t} f\right)(s):=f(s-t), \quad s, t \in \mathbb{R}, f \in X .
\end{gathered}
$$

Again we take $w(t)=1$ for $t \in \mathbb{R}$. The Phillips calculus is

$$
(\widehat{\mu}(A) f)(s)=\int_{\mathbb{R}} f(s-t) d \mu(t)=\left(L_{\mu} f\right)(s) .
$$

One can directly compute:

$$
\sigma(A)=i \mathbb{R}, \quad \sigma\left(e^{A t}\right)=\{\lambda \in \mathbb{C} ;|\lambda|=1\},
$$

so

$$
\begin{aligned}
& e^{t \sigma(A)}=\{\lambda \in \mathbb{C} ;|\lambda|=1\}=\sigma\left(e^{A t}\right), \\
& \sigma(\widehat{\mu}(A))=\sigma\left(L_{\mu}\right), \\
& \widehat{\mu}(\sigma(A))=\widehat{\mu}(i \mathbb{R})=\widehat{\mu}(\Delta X) .
\end{aligned}
$$

We see that WSMP holds for the Dirac measures $\mu=\delta_{t_{0}}$ and in general, WSMP holds for $\mu$ if and only if $\mu$ has natural spectrum. However, it is known that if $\mu \in M(\mathbb{R})$ has a decomposition $\mu=\mu_{\mathrm{ac}}+\mu_{\mathrm{at}}+\mu_{\mathrm{s}}$ (into absolutely continuous, atomic and non-atomic singular parts) with $\mu_{\mathrm{s}}=0$ then $\mu$ has natural spectrum, and that there exist $\mu$ without natural spectrum (whenever the Wiener-Pitt effect on $\mathbb{R}$ holds for $\mu$ ). For the details see [5, p. 371, Theorem 4.11.5].

Acknowledgements. The authors would like to thank the referee for his careful reading and for comments improving the paper.

\section{References}

[1] C. D'Antoni, R. Longo and L. Zsidó, A spectral mapping theorem for locally compact groups of operators, Pacific J. Math. 103 (1981), 17-24. 
[2] E. B. Davies, One-Parameter Semigroups, London Math. Soc. Monogr. 15, Academic Press, London, 1980.

[3] K.-J. Engel and R. Nagel, One-Parameter Semigroups for Linear Evolution Equations, Springer, 2000.

[4] E. Hille and R. S. Phillips, Functional Analysis and Semi-Groups, Amer. Math. Soc., Providence, 1957.

[5] K. B. Laursen and M. M. Neumann, An Introduction to the Local Spectral Theory, Clarendon Press, Oxford, 2000.

[6] R. Nagel and S.-Z. Huang, Spectral mapping theorems for $\mathcal{C}_{0}$-groups satisfying nonquasianalytic growth conditions, Math. Nachr. 169 (1994), 207-218.

[7] A. M. Sinclair, Continuous Semigroups in Banach Algebras, Cambridge Univ. Press, 1982.

[8] J. H. Williamson, The Wiener-Pitt phenomenon on the half-line, Proc. Edinburgh Math. Soc. 13 (1962), 37-38.

Department of Mathematical Analysis

Charles University

Sokolovská 83

18675 Praha, Czech Republic

E-mail: fasanga@karlin.mff.cuni.cz
Department of Mathematics Universidad de Zaragoza 50009 Zaragoza, Spain E-mail:pjmiana@posta.unizar.es

Received May 8, 2003

Revised version April 2, 2004 